\title{
Elementary Wave Interactions for the Simplified Combustion Model in Magnetogasdynamics
}

\author{
Yujin Liu*, Wenhua Sun \\ School of Mathematics and Statistics, Shandong University of Technology, Zibo, China \\ Email: *yjliu98@126.com
}

How to cite this paper: Liu, Y.J. and Sun, W.H. (2019) Elementary Wave Interactions for the Simplified Combustion Model in Magnetogasdynamics. Journal of Applied Mathematics and Physics, 7, 983-1000. https://doi.org/10.4236/jamp.2019.74066

Received: March 22, 2019

Accepted: April 26, 2019

Published: April 29, 2019

Copyright (อ 2019 by author(s) and Scientific Research Publishing Inc. This work is licensed under the Creative Commons Attribution International License (CC BY 4.0).

http://creativecommons.org/licenses/by/4.0/

\begin{abstract}
We investigate the elementary wave interactions for the simplified combustion model in magnetogasdynamics. Under the modified entropy conditions, we construct the unique solution and observe some interesting phenomena; such as, the combustion wave may be extinguished by the contact discontinuity or the shock wave. Especially, the transition between the detonation wave and the deflagration wave is also captured.
\end{abstract}

\section{Keywords}

Riemann Problem, Wave Interaction, Hyperbolic Conservation Laws, Combustion, Magnetogasdynamics

\section{Introduction}

Magnetogasdynamics is very important in studying engineering physics [1]-[9]. It is difficult to investigate the governing equations of Magnetogasdynamics flows; the corresponding results are less than the conventional gas dynamics. When the velocity field and the magnetic field are everywhere orthogonal, the magnetogasdynamics flow is still important.

In [3], Helliwell discussed the non-conducting inviscid gas at rest when ionization of the gas takes place across a shock wave and obtained that the magnetogasdynamic combustion wave has similar properties with the conventional gas dynamics. In [6], Mareev investigated the above problem further and they could use the obtained results to study the hypersonic gas flow around a thin wedge in an axial magnetic field.

In [4], $\mathrm{Hu}$ and Sheng constructed the unique Riemann solution of the one-dimensional inviscid flow 


$$
\left\{\begin{array}{l}
\tau_{t}-u_{x}=0, \\
u_{t}+\left(p+\frac{B^{2}}{2 \mu}\right)_{x}=0, \\
\left(E+\frac{B^{2} \tau}{2 \mu}\right)_{t}+\left(p u+\frac{B^{2} u}{2 \mu}\right)_{x}=0,
\end{array}\right.
$$

under the assumption $B=k \rho$, where $\tau>0, p \geq 0, u, B \geq 0$ and $\mu$ are respectively the specific volume, pressure, velocity, transverse magnetic field and magnetic permeability. $E=e+\frac{u^{2}}{2}$ is the specific total energy. and $e$ is the specific internal energy. The Riemann problem of the conventional gas dynamics combustion models was investigated by many people ([10] [11] [12] [13], etc.). Zhang and Zheng [13] studied the Riemann problem of the conventional gas dynamics flow of combustible ideal gases

$$
\left\{\begin{array}{l}
u_{t}+p_{x}=0, \\
\tau_{t}-u_{x}=0, \\
E_{t}+(u p)_{x}=0,
\end{array}\right.
$$

with an infinite rate of reaction which is described by

$$
q(x, t)= \begin{cases}0, & \text { if } \sup _{0 \leq y \leq t} T(x, y)>T_{i} ; \\ q(x, 0), & \text { otherwise. }\end{cases}
$$

Under the proposed global entropy conditions, they constructed uniquely the Riemann solutions by the characteristic analysis. In [11], we modified the above global entropy conditions and constructed the unique solution of the generalized Riemann problem for (2) and (3).

In [5], we obtained uniquely the Riemann solutions for (1) and (3) under the modified entropy conditions in [11] with the following initial data

$$
(\tau, p, u, q)(x, 0)=\left(\tau^{ \pm}, p^{ \pm}, u^{ \pm}, q^{ \pm}\right), \pm x>0,
$$

where $\tau^{ \pm}>0, p^{ \pm}, u^{ \pm}$are arbitrary constants,

$$
q^{ \pm}= \begin{cases}0, & \text { if } T^{ \pm}>T_{i}, \\ 0 \text { or } q_{0}, & \text { if } T^{ \pm} \leq T_{i},\end{cases}
$$

and $q_{0}>0$ is a constant. $E=e+\frac{u^{2}}{2}+q$ is the specific total energy, where $q$ is the chemical binding energy. The temperature $T$ satisfies Boyle and Gay-Lussac's law: $p \tau=R T . T_{i}$ is the ignition temperature. For polytropic gases, we know $e=e(T)$ and $E=\frac{u^{2}}{2}+\frac{p \tau}{\gamma-1}+q$, where $\gamma>1$ is the adiabatic exponent. For simplicity, we usually assume that $R$ and $\gamma$ remain unchanged during the reaction. We also assume that the combustion process is exothermic, i.e. the energy used up in recombing the atoms to form the new molecules is smaller for the burnt gas than the binding energy of the unburnt gas [10]. 
In the present paper, we are concerned with the wave interactions of the elementary waves of the Chapman-Jouguet (CJ) model (1) and (3). we can capture some interesting combustion phenomena by investigating the elementary wave interactions. For example, in most cases expect for Case 5. In Section 3, the combustion wave may be extinguished by the contact discontinuity or the shock wave. Especially, a detonation wave may be transformed into a deflagration wave by the contact discontinuity (see Case 1 and Case 2 in Section 3) and a deflagration wave may be transformed into a detonation wave by the contact discontinuity (see Case 4 in Section 3) or by the shock wave (see Case 6 in Section 3) which shows the transition between the detonation wave and the deflagration wave. In the case that when combustion waves interact with the contact discontinuity, our results are very different from the conventional gas dynamics combustion model (2) and (3) where there is no transition from the detonation (deflagration) wave to the deflagration (detonation) wave and the combustion wave can not be extinguished by the contact discontinuity.

This paper is organized as follows. In Section 2, we present the results of the Riemann problem for the CJ model (1), (3) with the initial values (4). In Section 3 , the elementary wave interactions are considered case by case under the modified entropy conditions in [11].

\section{Preliminaries}

As a preparation, we study the Riemann problem for the CJ model (1), (3) with the initial data (4) and we refer the detailed discussions to [4] [5].

There are three eigenvalues of (1) which are $\lambda_{1}=-\left(\frac{p-e_{p} \frac{B B_{\tau}}{\mu}+e_{\tau}}{e_{p}}\right)^{\frac{1}{2}}$,

$\lambda_{2}=0$ and $\lambda_{3}=\left(\frac{p-e_{p} \frac{B B_{\tau}}{\mu}+e_{\tau}}{e_{p}}\right)^{\frac{1}{2}}$. If $e_{p}>0$ and $e_{\tau}+p>0,(1)$ is strictly

hyperbolic. The characteristic fields $\lambda_{1,3}$ are genuinely nonlinear and $\lambda_{2}$ is linearly degenerate.

Considering the self-similar solution $(\tau, p, u)(\zeta)\left(\zeta=\frac{x}{t}\right)$, for any smooth solution we have

$$
\left\{\begin{array}{l}
\zeta \mathrm{d} \tau=-\mathrm{d} u \\
\zeta \mathrm{d} u=\mathrm{d}\left(p+\frac{B^{2} u}{2 \mu}\right), \\
\zeta \mathrm{d}\left(E+\frac{B^{2} u}{2 \mu} \tau\right)=\mathrm{d}\left(u p+\frac{B^{2} u}{2 \mu} u\right) .
\end{array}\right.
$$

The forward or backward rarefaction waves $\vec{R}$ passing through the point $\left(\tau_{0}, p_{0}, u_{0}\right)$ are 


$$
\left\{\begin{array}{l}
p \tau^{\gamma}=p_{0} \tau_{0}^{\gamma}, \\
u=u_{0} \pm \int_{p_{0}}^{p} \frac{\sqrt{\gamma p \tau+\frac{B^{2} \tau}{\mu}}}{\gamma p} \mathrm{~d} p .
\end{array}\right.
$$

The Rankine-Hugoniot jump conditions at $\zeta=\sigma$ are as follows

$$
\left\{\begin{array}{l}
\sigma[\tau]=-[u] \\
\sigma[u]=\left[p+\frac{B^{2} u}{2 \mu}\right] \\
\sigma\left[E+\frac{B^{2} u}{2 \mu} \tau\right]=\left[u p+\frac{B^{2} u}{2 \mu} u\right]
\end{array}\right.
$$

where $[\tau]=\tau_{r}-\tau_{l}$, etc.

The contact discontinuity $J$ is given by

$$
[u]=\left[p+\frac{B^{2}}{2 \mu}\right]=0,
$$

and $J$ is a curve in the $(\tau, p, u)$ space and the projection on the $(p, u)$ plane is a straight line parallel to the $p$-axis. Denote $J$ by $J$ when $p_{l}<p_{r}, \tau_{l}<\tau_{r}$, and $\vec{J}$ when $p_{l}>p_{r}, \tau_{l}>\tau_{r}$.

If $[q]=0$ in (7), we get the forward or backward shock waves $\overrightarrow{\vec{S}}$ passing through the point $\left(\tau_{0}, p_{0}, u_{0}\right)$

$$
\left\{\begin{array}{l}
\left(p+\theta^{2} p_{0}+\theta^{2}\left(\frac{3 B^{2}}{2 \mu}+\frac{B_{0}^{2}}{2 \mu}\right)\right) \tau=\left(p_{0}+\theta^{2} p+\theta^{2}\left(\frac{3 B_{0}^{2}}{2 \mu}+\frac{B^{2}}{2 \mu}\right)\right) \tau_{0}, \\
u=u_{0} \pm\left(p+\frac{B^{2}}{2 \mu}-p_{0}-\frac{B_{0}^{2}}{2 \mu}\right) \sqrt{-\frac{\tau-\tau_{0}}{p+\frac{B^{2}}{2 \mu}-p_{0}-\frac{B_{0}^{2}}{2 \mu}}}
\end{array}\right.
$$

where $\theta^{2}=\frac{\gamma-1}{\gamma+1}$ and $B_{0}=\frac{k}{\tau_{0}}$.

If $[q] \neq 0$ in (7), we obtain the combustion wave curve in the $(\tau, p)$ plane

$$
\begin{aligned}
& D_{u}(0):\left(\tau-\theta^{2} \tau_{0}\right)\left(p+\theta^{2}\left(p_{0}+\frac{B_{0}^{2}}{2 \mu}+\frac{3 B^{2}}{2 \mu}\right)\right) \\
& =\left(1-\theta^{4}\right) \tau_{0} p_{0}+\frac{\theta^{2} \tau_{0}}{\mu}\left[B_{0}^{2}\left(3-\theta^{2}\right)+B^{2}\left(1-3 \theta^{2}\right)\right]+2 \theta^{2} q_{0} .
\end{aligned}
$$

Draw two straight lines from $\left(\tau_{0}, p_{0}\right)$ and they are tangent to the above curve. We call the tangent points $A$ with $\tau<\tau_{0}$ and $B$ with $\tau>\tau_{0}$ Chapman-Jouguet detonation (CJDT) and Chapman-Jouguet deflagration $(C J D F)$, respectively. From the RH condition (7), we should disregard the curve between the points $C$ and $D$. We call the curve between $C$ and $A$ weak detonation (WDT) and the curve above $A$ strong detonation (SDT), the curve between $D$ and $B$ weak deflagration $(W D F)$ and the curve below $B$ strong deflagration $(S D F)$, respectively (see Figure 1 ). 


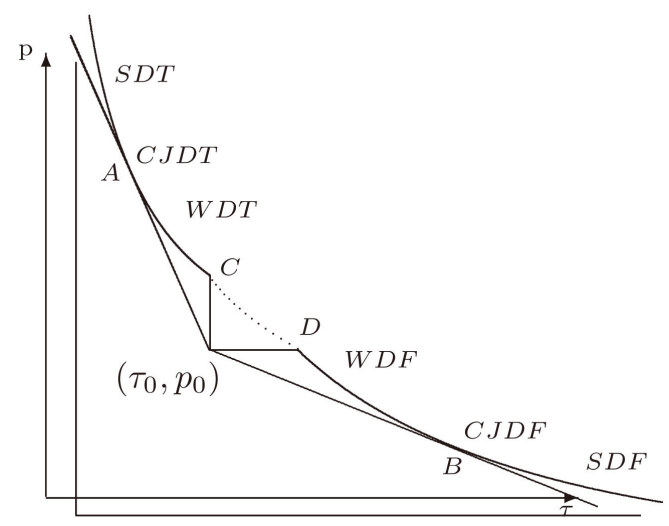

Figure 1. The combustion wave curve in the $(\tau, p)$.

From the known Jouguet's rule in [13], there are at most three different kinds of wave series that can be linked to the state $(l) \equiv\left(\tau_{l}, p_{l}, u_{l}, q_{l}\right)$ :

1) $S_{u}(l)$ or $R_{u}(l)$ (containing no combustion waves),

2) $(i)+W D F(i)$ or $(i)+C J D F(i)+R(C J D F(i))$ (containing no $D T$ waves),

3) $\operatorname{SDT}(l)$ or $C J D T(l)+R(C J D T(l))$ (containing no $D F$ waves), where $i \equiv i(l) \equiv\left(u_{i}, p_{i}, \tau_{i}, q\right)$ is the state at $S(l)$ with the ignition temperature $T_{i}$, and the symbol "+" means "followed by". Notice that we let the temperature behind the pre-compressive shock wave which connects the state $(I)$ and the ignition point $(i)$ be the ignition point $T_{i}$, we just need to construct the deflagration wave curve which is the successor to the pre-compressive shock wave from the point $(i)$.

In the $(\tau, p)$-plane we have

$$
\begin{gathered}
R_{u}(l): p \tau^{\gamma}=p_{-} \tau_{-}^{\gamma}, \quad\left(0<p<p_{l}\right), \\
S_{u}(l):\left(\tau-\theta^{2} \tau_{l}\right)\left(p+\theta^{2}\left(p_{l}+\frac{B_{l}^{2}}{2 \mu}+\frac{3 B^{2}}{2 \mu}\right)\right) \\
=\left(1-\theta^{4}\right) \tau_{l} p_{l}+\frac{\theta^{2} \tau_{l}}{\mu}\left[B_{l}^{2}\left(3-\theta^{2}\right)+B^{2}\left(\left(1-3 \theta^{2}\right)\right], \quad\left(p>p_{l}\right),\right. \\
\operatorname{SDT}(l):\left(\tau-\theta^{2} \tau_{l}\right)\left(p+\theta^{2}\left(p_{l}+\frac{B_{l}^{2}}{2 \mu}+\frac{3 B^{2}}{2 \mu}\right)\right) \\
=\left(1-\theta^{4}\right) \tau_{l} p_{l}+\frac{\theta^{2} \tau_{l}}{\mu}\left[B_{l}^{2}\left(3-\theta^{2}\right)+B^{2}\left(1-3 \theta^{2}\right)\right]+2 \theta^{2} q_{0}, \quad\left(p>p_{A}\right), \\
W D F(i):\left(\tau-\theta^{2} \tau_{i}\right)\left(p+\theta^{2}\left(p_{i}+\frac{B_{i}^{2}}{2 \mu}+\frac{3 B^{2}}{2 \mu}\right)\right) \\
=\left(1-\theta^{4}\right) \tau_{i} p_{i}+\frac{\theta^{2} \tau_{i}}{\mu}\left[B_{i}^{2}\left(3-\theta^{2}\right)+B^{2}\left(1-3 \theta^{2}\right)\right]+2 \theta^{2} q_{0},\left(\left(p_{D}\right)_{i}<p<p_{i}\right), \\
R(C J D T(l)): p \tau^{\gamma}=p_{A} \tau_{C}^{\gamma}, \quad\left(p<p_{A}\right), \\
R(C J D F(l)): p \tau^{\gamma}=\left(p_{B}\right)_{i}\left(\tau_{B}\right)_{i}^{\gamma}, \quad\left(p<\left(p_{B}\right)_{i}\right) .
\end{gathered}
$$

Denote $W(l):=W_{S}(l) \cup W_{D}(l)$, where $W_{S}(l)$ denotes $\left(W_{S}(l), q_{l}=0\right)$ or 
$\left(W_{S}(l), q_{l}>0\right)$ or both of them, and $W_{D}(l)$ denotes $W_{D T}(l) \cup W_{D F}(l)$, here $W_{D T}(l):=S D T(l) \cup C J D T(l) \cup R(C J D T(l))$ and

$W_{D F}(l):=W D F(i) \cup C J D F(i) \cup R(C J D T(i))$.

Now we study the combustion wave curves in the $(u, p)$ plane and construct the backward combustion wave curve $\bar{W}(l)$ from the state $(l)=\left(\tau_{l}, p_{l}, u_{l}, q_{l}\right)$.

From (9), for the backward wave $\bar{S}_{\tau}\left(Q_{0 \tau}\right)$ we get

$$
u=u_{0}-\sqrt{\left(p+\frac{B^{2}}{2 \mu}-p_{0}-\frac{B_{0}^{2}}{2 \mu}\right)\left(\tau_{0}-\tau\right)}, u<u_{0},
$$

and from (10), we know

$$
\tau=\frac{\left(1-\theta^{4}\right) \tau_{0} p_{0}+\frac{\theta^{2} \tau_{0}}{\mu}\left[B_{0}^{2}\left(3-\theta^{2}\right)+B^{2}\left(1-3 \theta^{2}\right)\right]+2 \theta^{2} q_{0}}{p+\theta^{2}\left(p_{0}+\frac{B_{0}^{2}}{2 \mu}+\frac{3 B^{2}}{2 \mu}\right)}+\theta^{2} \tau_{0},
$$

and

$$
\tau-\tau_{0}=\frac{\left(1-\theta^{2}\right) \tau_{0}\left(p_{0}-p\right)+\frac{\theta^{2} \tau_{0}}{\mu}\left(B_{0}^{2}-B^{2}\right)+2 \theta^{2} q_{0}}{p+\theta^{2}\left(p_{0}+\frac{B_{0}^{2}}{2 \mu}+\frac{3 B^{2}}{2 \mu}\right)},
$$

thus we obtain the backward combustion wave curve in the $(u, p)$ plane (see Figure 2).

$$
\begin{aligned}
& \bar{D}_{\tau}(0): u \\
& =u_{0}-\sqrt{\left(p+\frac{B^{2}}{2 \mu}-p_{0}-\frac{B_{0}^{2}}{2 \mu}\right) \frac{\left(1-\theta^{2}\right) \tau_{0}\left(p-p_{0}\right)+\frac{\theta^{2} \tau_{0}}{\mu}\left(B^{2}-B_{0}^{2}\right)-2 \theta^{2} q_{0}}{p+\theta^{2}\left(p_{0}+\frac{B_{0}^{2}}{2 \mu}+\frac{3 B^{2}}{2 \mu}\right)}} .
\end{aligned}
$$

Now denote the backward $D F$ and $D T$ wave curve by $\bar{W}_{D F}(l)$ and $\bar{W}_{D T}(l)$, respectively, where

$$
\begin{aligned}
\bar{W}_{D F}(l) & :=\overline{W D F}\left(i_{s}\right) \cup \overline{C J D F}\left(i_{s}\right) \cup \bar{R}\left(\overline{C J D F}\left(i_{s}\right)\right), \\
\bar{W}_{D T}(l) & :=\overline{S D T}(l) \cup \overline{C J D T}(l) \cup \bar{R}(\overline{C J D T}(l)) .
\end{aligned}
$$

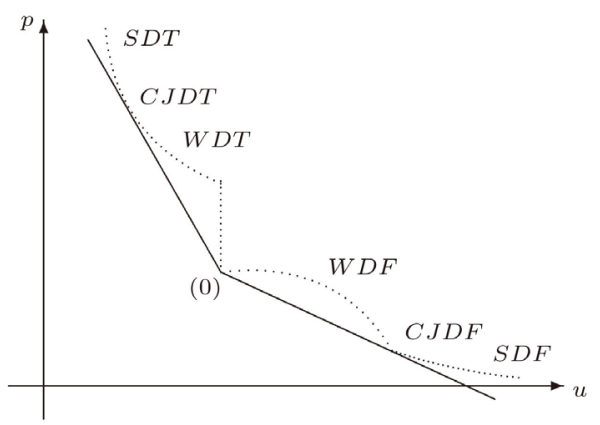

Figure 2. The combustion wave curve in the plane $(u, p)$. 
Denote the backward wave curve $\bar{W}(l)$ which can be linked to the state $(l)=\left(\tau_{l}, p_{l}, u_{l}, q_{l}\right)$, then

$$
\begin{gathered}
\bar{W}(l):=\bar{W}_{S}(l) \cup \bar{W}_{D}(l), \\
\bar{W}_{S}(l):=\left(\bar{W}_{S}(l), q_{l}=0\right) \operatorname{or}\left(\bar{W}_{S}(l), q_{l}>0\right), \\
\bar{W}_{D}(l):=\bar{W}_{D F}(l) \cup \bar{W}_{D T}(l) .
\end{gathered}
$$

Similarly, we can construct the forward wave curve $\vec{W}(r)$ that can be linked to the state $(r)=\left(\tau_{r}, p_{r}, u_{r}, q_{r}\right)$.

Since the image of $\mathrm{J}$ in $(\tau, p, u)$ is a straight line which parallels to the $\tau$-axis and the projection on the plane $(u, p)$ is a point, $J$ is a plane curve in $(\tau, p, u)$ and the projection on the plane $(u, p)$ is a straight line which parallels to the $p$-axis. Thus the Riemann prblem for (1) is much more complicated than that of the conventional gas dynamics.

When $q_{l}=q_{r}=0$, the gas on both sides are burnt, no combustion wave will occur.

When $q_{l}$ and $q_{r}$ are not both zero, there may exist more than one intersection points of $\bar{W}(l)$ and $\vec{W}(r)$. Each intersection point corresponds to a unique Riemann solution. When the intersection point is unique, the solution is also unique, otherwise, in order to obtain the unique solution we select it under the following modified global entropy conditions (MGEC) ([11]):

We select the unique solution from the nine intersection points (at most) of the forward wave curves connecting $(r)$ and the backward wave curves connecting $(I)$ in the following order:

1) the solution with the propagating speed of combustion wave as low as possible;

2) the solution with the parameter $\beta$ as small as possible, where $\beta$ is defined as oscillation frequency of $T(\xi)$ between the set $\left\{\xi \in R^{1}: T(\xi) \leq T_{i}\right\}$ and the set $\left\{\xi \in R^{1}: T(\xi)>T_{i}\right\}$;

3) the solution containing as many combustion wave as possible.

Case 1. $q_{l}>0, q_{r}=0$. In this case, the gas is unburnt on the left side, the gas is burnt on the right side, i.e. $\bar{W}(l)=\bar{W}_{S}(l) \cup \bar{W}_{D F}(l) \cup \bar{W}_{D T}(l), \quad \vec{W}(r)=\vec{W}_{S}(r)$. When there exists only one intersection point of $\bar{W}(l)$ and $\vec{W}(r)$, we obtain the unique solution is a detonation wave solution $\overline{D T}+\vec{R}$ or $\vec{S}$ if $p_{l} \tau_{l}^{\gamma}=p_{r} \tau_{r}^{\gamma}$, or $\overline{D T}+J+\vec{R}$ or $\vec{S}$ if $p_{l} \tau_{l}^{\gamma} \neq p_{r} \tau_{r}^{\gamma}$.

When there are three intersection points of $\bar{W}(l)$ and $\vec{W}_{S}(r)$ (see Figure 3 ), from the modified global entropy condition $A$, we discard the intersection point of $\vec{W}_{S}(r)$ and $\bar{W}_{D T}(l)$. Denote the intersection point of $\vec{W}_{S}(r)$ and $\bar{W}_{S}(l)$ by $*_{S}$ and the intersection point of $\vec{W}_{S}(r)$ and $\bar{W}_{D F}(l)$ by $*_{D F}$. Denote the temperature at the point $*_{S}, *_{D F}$ on $\vec{W}_{S}(r)$ by $T_{S}, T_{D F}$, respectively. The temperature at $*_{D F}$ on $\bar{W}_{D F}(l)$ is greater than $T_{i}$ since the combustion process is exothermic.

Subcase 1.1. $p_{l} \tau_{l}^{\gamma}=p_{r} \tau_{r}^{\gamma}$. 


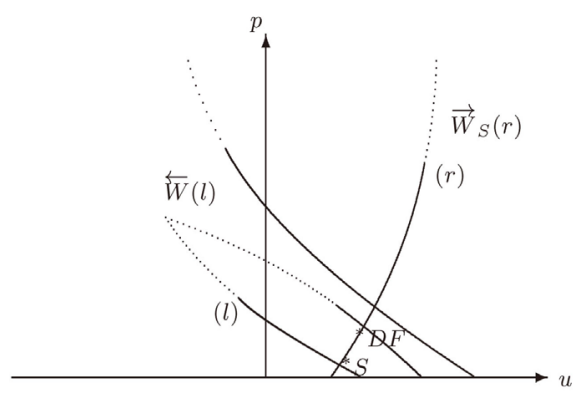

Figure 3. $q_{l}>0, q_{r}=0$ and there are there interactions.

When $T_{r} \leq T_{i}$, then $\beta\left(*_{S}\right)=0, \beta\left(*_{D F}\right)=2$, from the condition $B$, we select $*_{S}$ and obtain a non-combustion wave solution $\bar{R}$ or $\bar{S}+\vec{R}$ or $\vec{S}$ (Figure $4(\mathrm{a}))$.

When $T_{r}>T_{i}$, then $\beta\left(*_{s}\right)=1, \beta\left(*_{D F}\right)=1$, from the condition $C$, we select $*_{D F}$ and obtain a combustion wave solution $\overleftarrow{D T}+\vec{R}$ or $\vec{S}$ (Figure 4(b)).

Subcase 1.2. $p_{l} \tau_{l}^{\gamma} \neq p_{r} \tau_{r}^{\gamma}$.

From the condition $A$, we discard the possible detonation $D T$ wave solution and find that the possible Riemann solution is $\bar{R}$ or $\bar{S}+J+\vec{R}$ or $\vec{S}$ or $\overleftarrow{D T}+J+\vec{R}$ or $\vec{S}$. According to the modified global entropy conditions we obtain the unique Riemann solution as follows (see Figure 5 ).

1) When $T_{r}>T_{i}, T_{D F}>T_{i}$, then $\beta\left(*_{S}\right)=1, \beta\left(*_{D F}\right)=1$, from the condition $C$, we select $*_{D F}$ and obtain a combustion wave solution containing a $D F$ (Figure 5(b)).

2) When $T_{r}>T_{i}, T_{D F} \leq T_{i}\left(\Rightarrow T_{S} \leq T_{i}\right)$, then $\beta\left(*_{S}\right)=1, \beta\left(*_{D F}\right)=3$, from the condition $B$, we select $*_{S}$ and obtain a non-combustion wave solution (Figure 5(a)).

3) When $T_{r} \leq T_{i}, T_{S} \leq T_{i}$, then $\beta\left(*_{S}\right)=0, \beta\left(*_{D F}\right)=2$, from the condition $B$, we select $*_{s}$ and obtain a non-combustion wave solution (Figure 5(a)).

4) When $T_{r} \leq T_{i}, T_{S}>T_{i}\left(\Rightarrow T_{D F}>T_{i}\right)$, then $\beta\left(*_{S}\right)=2, \beta\left(*_{D F}\right)=2$, from the condition $C$, we select $*_{D F}$ and obtain a combustion wave solution containing a $D F$ (Figure $5(\mathrm{~b}))$.

Case 2. $q_{l}>0, q_{r}=0$ and there are two intersection points of $\bar{W}(l)$ and $\vec{W}_{S}(r)$ (see Figure 6).

Subcase 2.1. $p_{l} \tau_{l}^{\gamma}=p_{r} \tau_{r}^{\gamma}$.

In this case, we select the point $*_{S}$ or $*_{D T}$ and obtain the possible solutions $\bar{S}+\vec{R}$ or $\vec{S}$ or $\overleftarrow{D T}+\vec{R}$ or $\vec{S}$. Now we select the unique Riemann solution as follows.

When $T_{r}>T_{i}$, then $\beta\left(*_{S}\right)=1, \beta\left(*_{D T}\right)=1$, from the condition $C$, we select $*_{D T}$ and obtain a combustion wave solution $\overleftarrow{D T}+\vec{R}$ or $\vec{S}$ (Figure 7(a)).

When $T_{r} \leq T_{i}$, then $\beta\left(*_{S}\right)=0, \beta\left(*_{D T}\right)=2$, from the condition $B$, we select $*_{S}$ and obtain a non-combustion wave solution $\bar{S}+\vec{R}$ or $\vec{S}$ (Figure 7(b)).

Subcase 2.2. $p_{l} \tau_{l}^{\gamma} \neq p_{r} \tau_{r}^{\gamma}$.

There are two possibilities: one is that there exists only one intersection point of $\bar{W}(l)$ and $\vec{W}(r)$ and we obtain the unique Riemann solution is $\overleftarrow{D T}+\vec{J}+\vec{R}$ 


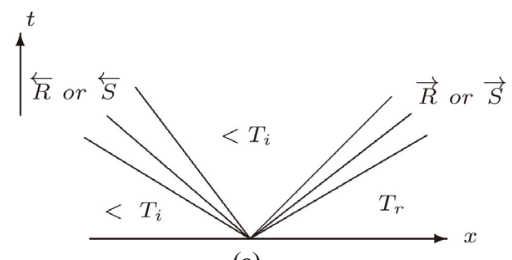

(a)

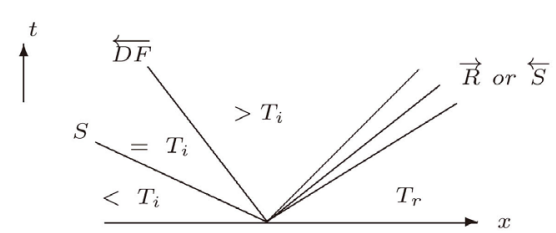

(b)

Figure 4. Solutions in Subcase 1.1. (a) non-combustion wave; (b) combustion wave DF.
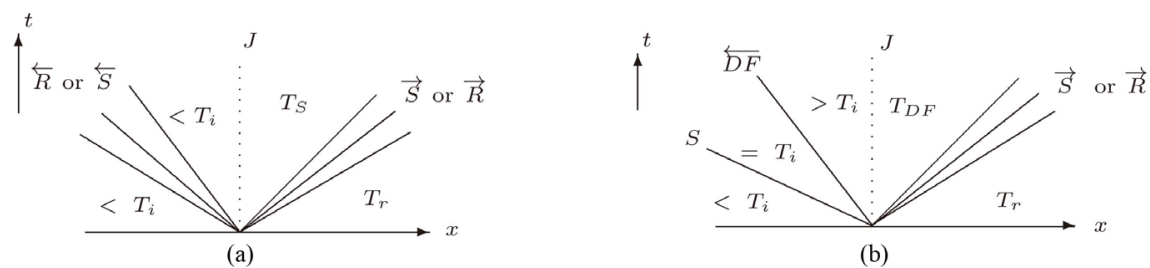

(b)

Figure 5. Solutions in Subcase 1.2. (a) non-combustion wave; (b) combustion wave DF.

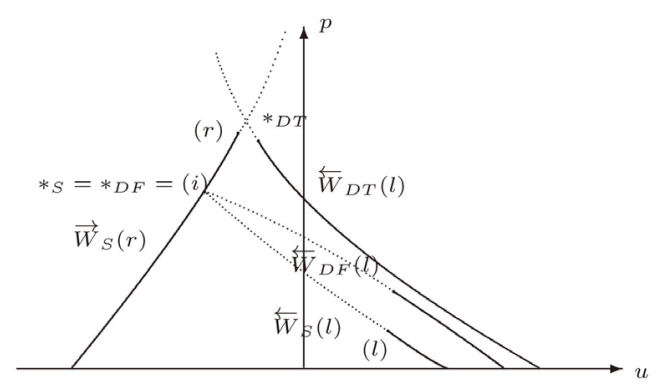

Figure 6. $q_{1}>0, q_{r}=0$ and there are two interactions.

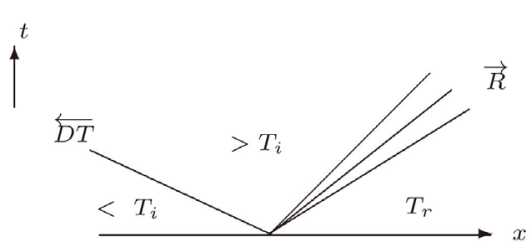

(a)

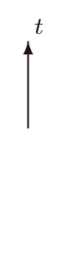

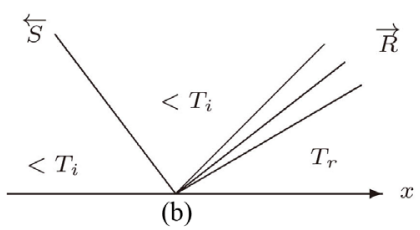

(b)

Figure 7. Solutions in Subcase 2.1. (a) combustion wave DT; (b) non-combustion wave.

or $\vec{S}$, the other one is that there are three possible solutions which are the noncombution wave solution $\bar{S}$ or $\bar{R}+J+\vec{R}$ or $\vec{S}$, or the $D F$ combustion wave solution $\overleftarrow{D T}+J+\vec{R}$ or $\vec{S}$, or the $D T$ combustion wave solution $\overleftarrow{D T}+\vec{J}+\vec{R}$ or $\vec{S}$. Similarly, according to the modified global entropy conditions we obtain the unique solution as follows (see Figure 8). From the global entropy condition $A$, we discard the $D T$ combustion wave solution.

1) When $T_{r}>T_{i}, T_{D F}>T_{i}$, then $\beta\left(*_{S}\right)=1, \beta\left(*_{D F}\right)=1$, from the condition $C$, we select $*_{D F}$ and obtain a combustion wave solution containing a $D F$ (Figure 8(b)).

2) When $T_{r}>T_{i}, T_{D F} \leq T_{i}\left(\Rightarrow T_{S} \leq T_{i}\right)$, then $\beta\left(*_{S}\right)=1, \beta\left(*_{D F}\right)=3$, from the condition $B$, we select $*_{s}$ and obtain a non-combustion wave solution (Figure 8(a)). 

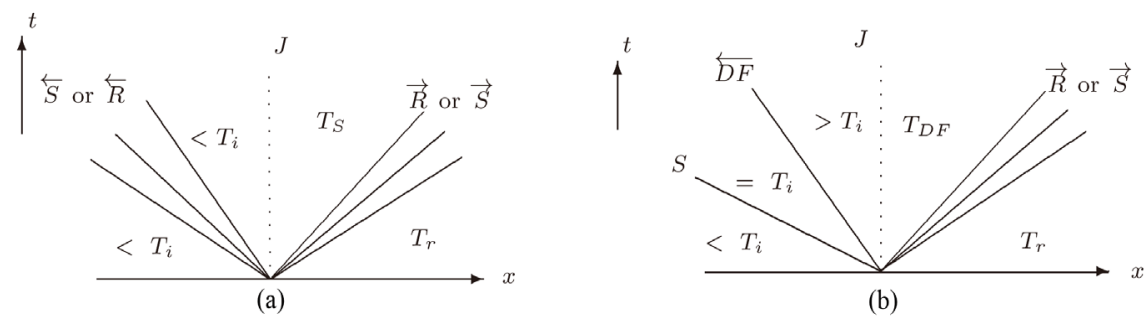

(b)

Figure 8. Solutions in Subcase 2.2. (a) non-combustion wave; (b) combustion wave DF.

3) When $T_{r} \leq T_{i}, T_{S} \leq T_{i}$, then $\beta\left(*_{S}\right)=0, \beta\left(*_{D F}\right)=2$, from the condition $B$, we select $*_{S}$ and obtain a non-combustion wave solution (Figure 8(a)).

4) When $T_{r} \leq T_{i}, T_{S}>T_{i}\left(\Rightarrow T_{D F}>T_{i}\right)$, then $\beta\left(*_{S}\right)=2, \beta\left(*_{D F}\right)=2$, from the condition $C$, we select $*_{D F}$ and obtain a combustion wave solution containing a $D F$ (Figure $8(\mathrm{~b})$ ).

Case 3. $q_{l}>0, q_{r}>0$ and the gas on the both sides are unburnt. In this case, we know that $\bar{W}(l)=\bar{W}_{S}(l) \cup \bar{W}_{D F}(l) \cup \bar{W}_{D T}(l)$, $\vec{W}(r)=\vec{W}_{S}(r) \cup \vec{W}_{D F}(r) \cup \vec{W}_{D T}(r)$.

If the intersection point of $\bar{W}(l)$ and $\vec{W}(r)$ is unique, the solution is $\overleftarrow{D T}+\overrightarrow{D T}$ if $p_{l} \tau_{l}^{\gamma}=p_{r} \tau_{r}^{\gamma}$, or $\overline{D T}+J+\overrightarrow{D T}$ if $p_{l} \tau_{l}^{\gamma} \neq p_{r} \tau_{r}^{\gamma}$. Otherwise, there are two possible subcases: one is that there is an intersection point of $\bar{W}_{S}(l)$ and $\vec{W}_{S}(r)$, the other is that there is no intersection point of $\bar{W}_{S}(l)$ and $\vec{W}_{S}(r)$.

Case 3.1. In the former subcase (see Figure 9), we discuss it in the following two subcases.

Subcase 3.1.1. $p_{l} \tau_{l}^{\gamma}=p_{r} \tau_{r}^{\gamma}$.

From the condition $A$, we just need to consider the intersection points 1, 2, 3, 4. We should select the unique solution from the four possible solutions (see Figure 10).

It is obvious that $\beta=0$ for (a), and it holds that $\beta=2$ for (b), (c) and (d). From the condition $B$, we select the intersection point 1 and obtain the unique non-combustion wave solution $\bar{R}$ or $\bar{S}+\vec{R}$ or $\vec{S}$.

Subcase 3.1.2. $p_{l} \tau_{l}^{\gamma} \neq p_{r} \tau_{r}^{\gamma}$. In a similar way as the above discussions in Subcase 3.1.1., we obtain that the unique Riemann solution is still the non-combustion wave solution $\bar{R}$ or $\bar{S}+J+\vec{R}$ or $\vec{S}$. The only difference is that here the contact discontinuity appears.

Case 3.2. In the latter subcase, there are only two possibilities: $\bar{W}(l)$ intersects $\vec{W}_{D T}(r)$ only or $\vec{W}(+)$ intersects $\bar{W}_{D T}(l)$ only. We just need to consider the former. If the intersection point is unique, the solution is $\overleftarrow{D T}+\overrightarrow{D T}$ if $p_{-} \tau_{-}^{\gamma}=p_{+} \tau_{+}^{\gamma}$, or $\overleftarrow{D T}+J+\overrightarrow{D T}$ if $p_{-} \tau_{-}^{\gamma} \neq p_{+} \tau_{+}^{\gamma}$, otherwise, there are at most three intersection points (see Figure 11).

Subcase 3.2.1. $p_{l} \tau_{l}^{\gamma} \neq p_{r} \tau_{r}^{\gamma}$.

From the condition $A$, the intersection point of $\bar{W}_{D T}(l)$ and $\vec{W}_{D T}(r)$ should be discarded. We denote the intersection point of $\vec{W}_{D T}(r)$ and $\bar{W}_{S}(l)$ by ${ }^{*} S$ and denote the intersection point of $\vec{W}_{D T}(+)$ and $\bar{W}_{D F}(l)$ by ${ }^{*} D F$, 


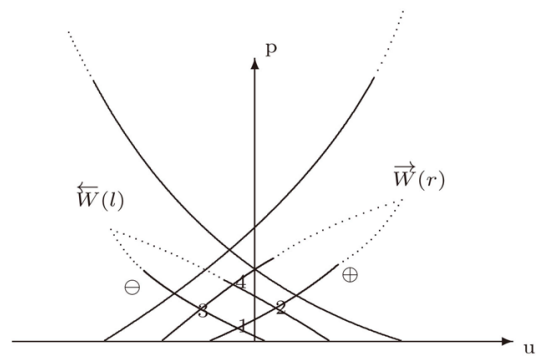

Figure 9. There is an intersection point of $\bar{W}_{S}(l)$ and $\vec{W}_{s}(r)$.

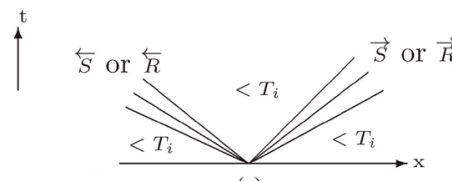

(a)

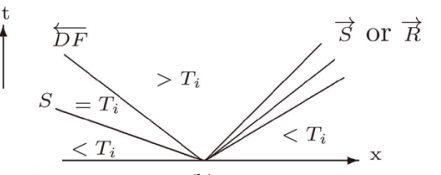

(b)
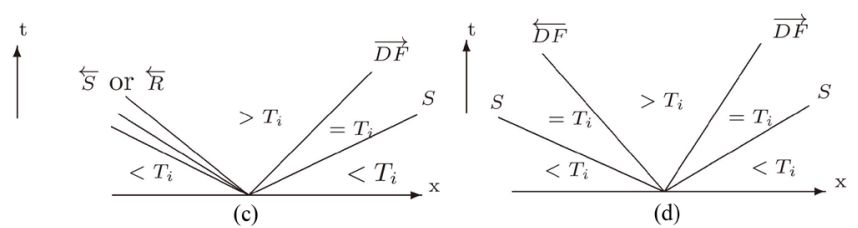

Figure 10. The possible solutions in Subcase 3.1.1. (a) solution corresponds to point 1; (b) solution corresponds to point 2; (c) solution corresponds to point 3; (d) solution corresponds to point 4 .

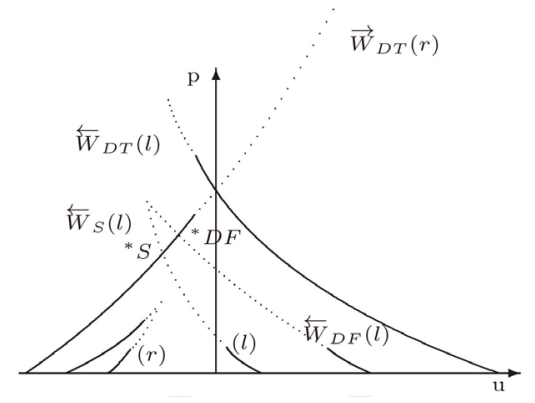

Figure 11. $\bar{W}(-)$ intersects $\vec{W}_{D T}(r)$ only.

respectively. We denote the temperature at the point ${ }^{*} S,{ }^{*} D F$ on $\vec{W}_{D T}(r)$ by $T_{S}, T_{D F}$, respectively (see Figure 12 ).

Since $T_{S}>T_{i}$ we have $T_{D F}>T_{i}$, then $\beta\left({ }^{*} S\right)=2, \beta\left({ }^{*} D F\right)=2$. From the condition $C$, we select ${ }^{*} D F$ and obtain a combustion wave solution $\overleftrightarrow{D F}+\overrightarrow{D T}$.

Subcase 3.2.2. $p_{l} \tau_{l}^{\gamma}=p_{r} \tau_{r}^{\gamma}$. In a similar way as the above discussions in Subcase 3.2.1., we obtain that the unique Riemann solution is still the combustion wave solution $\overleftarrow{D F}+\overrightarrow{D T}$. The only difference is that there exists the contact discontinuity in Subcase 3.2.1.

Based on the above analysis, we have the following result.

Theorem 2.1. There exists a unique piecewise smooth solution to the Riemann problem (1) and (3) with the initial data (4) under the modified global entropy conditions (MGEC). 

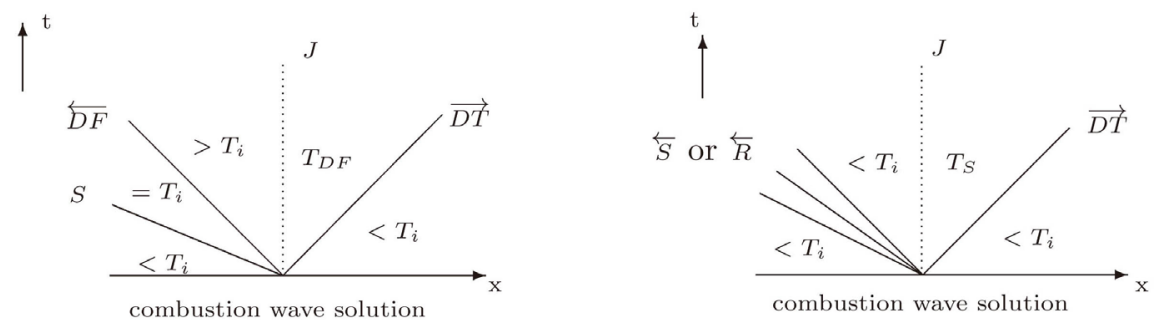

Figure 12. Solutions in Subcase 3.2.1.

\section{Wave Interactions for the Combustion Model (1) and (3)}

In this section, we discuss the wave interactions of the elementary waves for our combustion model (1) and (3). Consider the equations (1) and (3) with the following initial data

$$
(B, \rho, u)(x, 0)= \begin{cases}\left(B_{l}, \rho_{l}, u_{l}\right), & -\infty<x \leq x_{1}, \\ \left(B_{m}, \rho_{m}, u_{m}\right), & x_{1}<x \leq x_{2}, \\ \left(B_{r}, \rho_{r}, u_{r}\right), & x_{2}<x<\infty,\end{cases}
$$

for arbitrary $x_{1}, x_{2} \in R$.

In order to capture the interesting combustion phenomena, in the present paper we investigate the following kinds of interactions:

The interaction of a combustion wave $\overline{S D T}$ or $\overline{W D F}$ and a contact discontinuity: $\vec{J} \overline{S D T}, \stackrel{<}{J} \overline{S D T}, \vec{J} \overline{W D F}, \stackrel{<}{J W D F}$;

The interaction of a combustion wave $\overline{S D T}$ or $\overline{W D F}$ and a shock wave: $\overline{S D T} \bar{S}, \overline{W D F} \bar{S}$.

In what follows, we construct the solutions of the wave interactions case by case.

Case 1. $\vec{J} \overline{S D T}$

In the $(x, t)$ plane, $\vec{J}$ and $\overline{S D T}$ will encounter each other at a finite time and a new Riemann problem is formed with $(I)$ and $(r)$ as its left-hand side state and right-hand side state, respectively (Figure 13). We solve this new Riemann problem in the $(u, p)$ plane. It is obvious that $u_{l}=u_{m}>u_{r}, p_{l}>p_{m}$ and $\tau_{l}>\tau_{m}$.

From the analysis of the wave curves in the $(u, p)$ plane, we know that $\bar{W}(l)=\bar{W}_{S}(l) \cup \bar{W}_{D F}(l) \cup \bar{W}_{D T}(l)$, and $\vec{W}(r)=\vec{W}_{S}(r)$.

There are two possible cases: there is only one intersection point of $\bar{W}_{D T}(l)$ and $\vec{W}_{S}(r)$, or there are three intersection points of $\bar{W}(l)$ and $\vec{W}_{S}(r)$. From the arguments in the former section, we know the result is as follows:

When $p_{l} \tau_{l}^{\gamma}=p_{r} \tau_{r}^{\gamma}$, from the modified global entropy conditions we obtain that

$$
J \overline{S D T} \rightarrow\left\{\begin{array}{l}
\overline{S D T}+\vec{R} \text { or } \vec{S}, \\
\bar{S} \text { or } \bar{R}+\vec{R} \text { or } \vec{S}, \\
\overline{D F}+\vec{R} \text { or } \vec{S},
\end{array}\right.
$$

where " $\rightarrow$ " means the result of the wave interaction. 


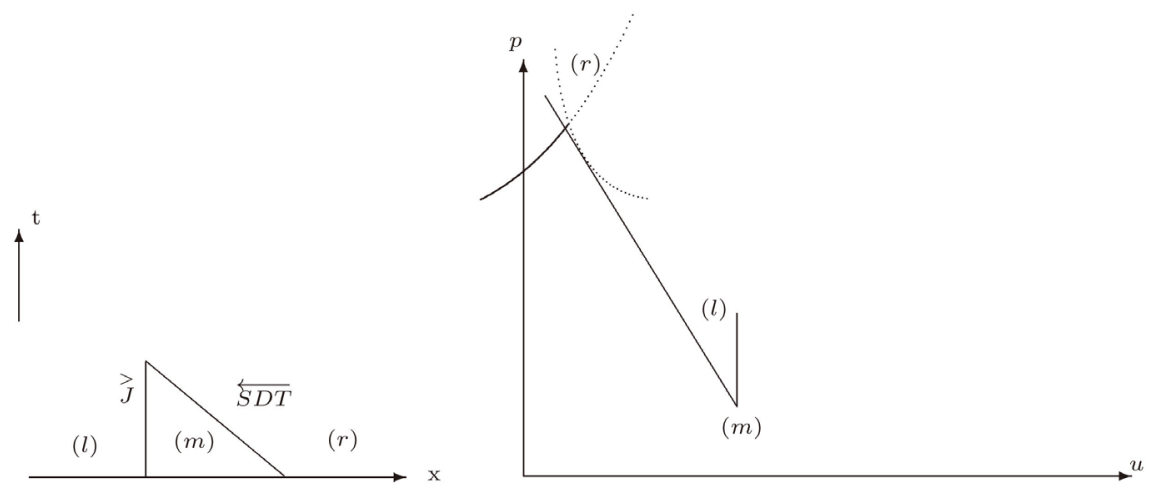

Figure 13. The interaction of $\vec{J}$ and $\overline{S D T}$.

When $p_{l} \tau_{l}^{\gamma} \neq p_{r} \tau_{r}^{\gamma}$, the results are given by

$$
\vec{J} \overleftarrow{S D T} \rightarrow\left\{\begin{array}{l}
\overleftarrow{S D T}+J+\vec{R} \text { or } \vec{S}, \\
\bar{S} \text { or } \overleftarrow{R}+J+\vec{R} \text { or } \vec{S}, \\
\overleftarrow{D F}+J+\vec{R} \text { or } \vec{S} .
\end{array}\right.
$$

Case 2. $\stackrel{<}{J} \overleftarrow{S D T}$

In this case (Figure 14), we know $u_{l}=u_{m}>u_{r}$, and for the contact discontinuity it holds that $p_{l}<p_{m}$ and $\tau_{l}<\tau_{m}$. Similar with the discussions as the above case, we know that when $p_{l} \tau_{l}^{\gamma}=p_{r} \tau_{r}^{\gamma}$,

$$
\left\langle\overleftarrow { J D T } \rightarrow \left\{\begin{array}{l}
\bar{S} \text { or } \bar{R}+\vec{R} \text { or } \vec{S}, \\
\overleftarrow{S D T}+\vec{R} \text { or } \vec{S}, \\
\overleftarrow{D F}+\vec{R} \text { or } \vec{S},
\end{array}\right.\right.
$$

when $p_{l} \tau_{l}^{\gamma} \neq p_{r} \tau_{r}^{\gamma}$, the results are the same as the above except that $J$ appears here.

Theorem 3.1. For Case 1 and Case 2, when a strong detonation combustion wave interacts with a contact discontinuity which is of a jump decrease (or increase) in density in the propagating direction of the strong detonation wave, the strong detonation combustion wave may be extinguished or cross the contact discontinuity at once, or be transformed into a deflagration wave. And the contact discontinuity may disappear or not.

Remark 3.2. The result of the interaction of the strong detonation wave and the contact discontinuity is very different from that of the corresponding case in the conventional gas dynamics combustion model (4) where there is no transition from the detonation wave to the deflagration wave and the combustion wave can not be extinguished by the contact discontinuity.

Case 3. $J \overline{W D F}$

For this case, we know that $\vec{J}$ and $\overline{W D F}$ will encounter each other at a finite time in the $(x, t)$ plane, and a new Riemann problem is formed with $(I)$ and $(r)$ as its left-hand side state and right-hand side state, respectively. Similarly, we solve the new Riemann problem in the $(u, p)$ plane. 


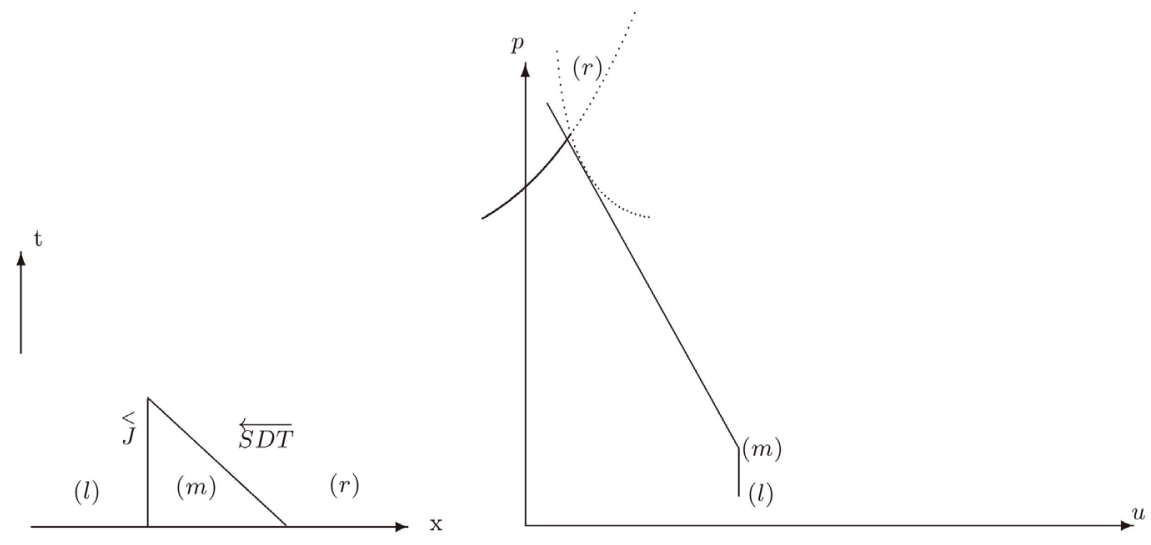

Figure 14. The interaction of $\stackrel{<}{J}$ and $\overline{S D T}$.

It is easily shown that $u_{l}=u_{m}$ and $p_{l}>p_{m}, \tau_{l}>\tau_{m}$. We proceed as follows (Figure 15). Based on the analysis in the $(u, p)$ plane, we notice specially that the temperature is just the ignition point on the wave front of the $\overline{W D F}$, i.e. the state $(m)=\left(i_{m}\right)$ where the symbol “ $\left(i_{m}\right)$ " means the ignition point corresponding to the state $(m)$. Since there are three intersection points of $\grave{W}(l)$ and $\vec{W}_{S}(r)$, from the global entropy conditions we know that when $p_{l} \tau_{l}^{\gamma}=p_{r} \tau_{r}^{\gamma}$,

$$
J \overline{W D F} \rightarrow\left\{\begin{array}{l}
\grave{S} \text { or } \bar{R}+\vec{R} \text { or } \vec{S}, \\
\overleftarrow{D F}+\vec{R} \text { or } \vec{S},
\end{array}\right.
$$

when $p_{l} \tau_{l}^{\gamma} \neq p_{r} \tau_{r}^{\gamma}$,

$$
J \overline{W D F} \rightarrow\left\{\begin{array}{l}
\overleftarrow{S} \text { or } \overleftarrow{R}+J+\vec{R} \text { or } \vec{S}, \\
\overleftarrow{D F}+J+\vec{R} \text { or } \vec{S} .
\end{array}\right.
$$

Theorem 3.3. For this case, when a weak deflagration combustion wave interacts with a contact discontinuity which is of a jump decrease in density in the propagating direction of the weak deflagration wave, the weak deflagration combustion wave may be extinguished, or cross the contact discontinuity at once. And the contact discontinuity may disappear or not.

Case 4. $J \overleftarrow{W D F}$

In this case (Figure 16), we know $u_{l}=u_{m}$, and for the contact discontinuity we know $p_{l}<p_{m}$ and $\tau_{l}<\tau_{m}$. There are two possibilities: one is that there is only one intersection point of $\bar{W}_{D T}(l)$ and $\vec{W}_{S}(r)$, the other one is that there are three intersection points of $\bar{W}(l)$ and $\vec{W}_{S}(r)$. Therefore we know the results are as follows:

When $p_{l} \tau_{l}^{\gamma}=p_{r} \tau_{r}^{\gamma}$, the results are as follows

$$
\dot{J} \overleftarrow{D F} \rightarrow\left\{\begin{array}{l}
\overleftarrow{D T}+\vec{R} \text { or } \vec{S}, \\
\overleftarrow{S} \text { or } \overleftarrow{R}+\vec{R} \text { or } \vec{S}, \\
\overleftarrow{D F}+\vec{R} \text { or } \vec{S},
\end{array}\right.
$$

when $p_{l} \tau_{l}^{\gamma} \neq p_{r} \tau_{r}^{\gamma}$, the results are the same as the above except that $J$ appears here. 


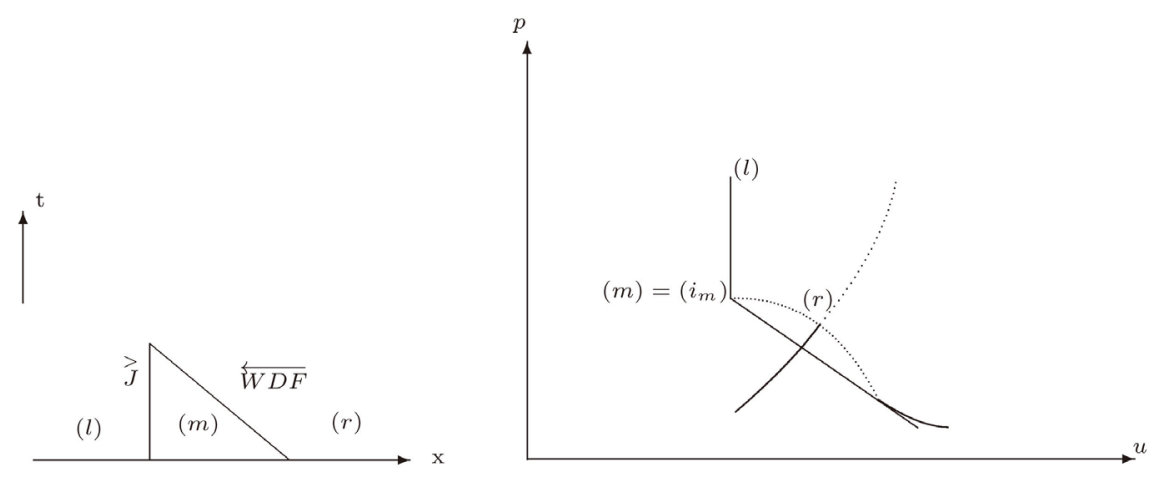

Figure 15. The interaction of $\vec{J}$ and $\overline{W D F}$.

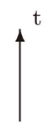

(l)

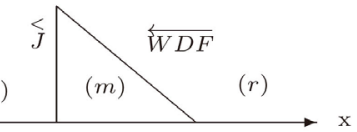

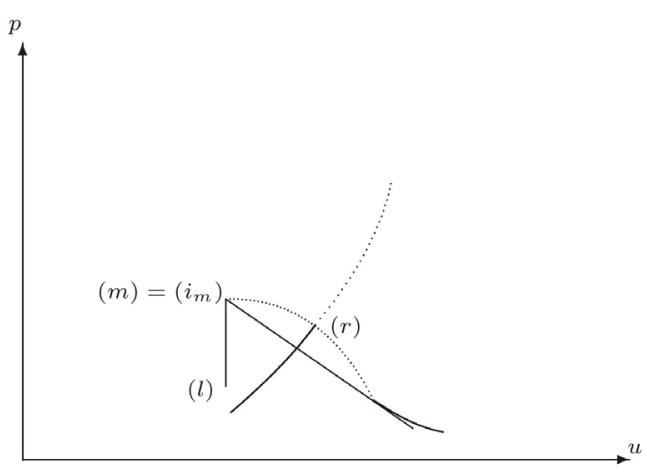

Figure 16. The interaction of $\stackrel{<}{J}$ and $\overline{W D F}$.

Theorem 3.4. For this case, when a weak deflagration combustion wave interacts with a contact discontinuity which is of a jump increase in density in the propagating direction of the weak deflagration wave, the weak deflagration combustion wave may be transformed into a detonation wave, or be extinguished, or cross the contact discontinuity at once. And the contact discontinuity may disappear or not.

Remark 3.5. The result of the interaction of the weak deflagration wave and the contact discontinuity is very different from that of the corresponding case in the conventional gas dynamics combustion model (2), where there is no transition from the deflagration wave to the detonation wave and the combustion wave can not be extinguished by the contact discontinuity.

Case 5. $\overline{S D T} \bar{S}$

In this case (Figure 17), we know for $\overline{S D T}$ it holds that $\lambda_{m}<\sigma_{1}<\lambda_{1}$, and for $\bar{S}$ it holds that $\lambda_{r}<\sigma_{2}<\lambda_{m}$, where $\sigma_{1}$ and $\sigma_{2}$ are respectively the velocity of $\overline{S D T}$ and $\bar{S}$. Therefore, $\bar{S}$ will overtake $\overline{S D T}$ at a finite time and a new Riemann problem is formed. We still solve this problem in the $(u, p)$ plane. $(m) \in \bar{W}_{D T}(l),(r) \in \bar{S}(m)$ and the curves $\bar{W}_{S}(l) \cup \bar{W}_{D F}(l)$ are located below the curve $\vec{W}_{S}(m)$ according to the condition $A$. From Lemma 2.6., we know $\bar{W}_{S}(l) \cup \bar{W}_{D F}(l)$ are located below $\vec{R}(m)\left(u \leq u_{m}\right)$ and the half straight line $p=p_{m}\left(u>u_{m}\right)$. Considering that $\vec{R}(m)$ and $\vec{R}(r)$ from Lemma 2.6., we know that $\vec{W}_{S}(r)$ is located above the curve $\vec{R}(m)\left(u \leq u_{m}\right)$ and the half 

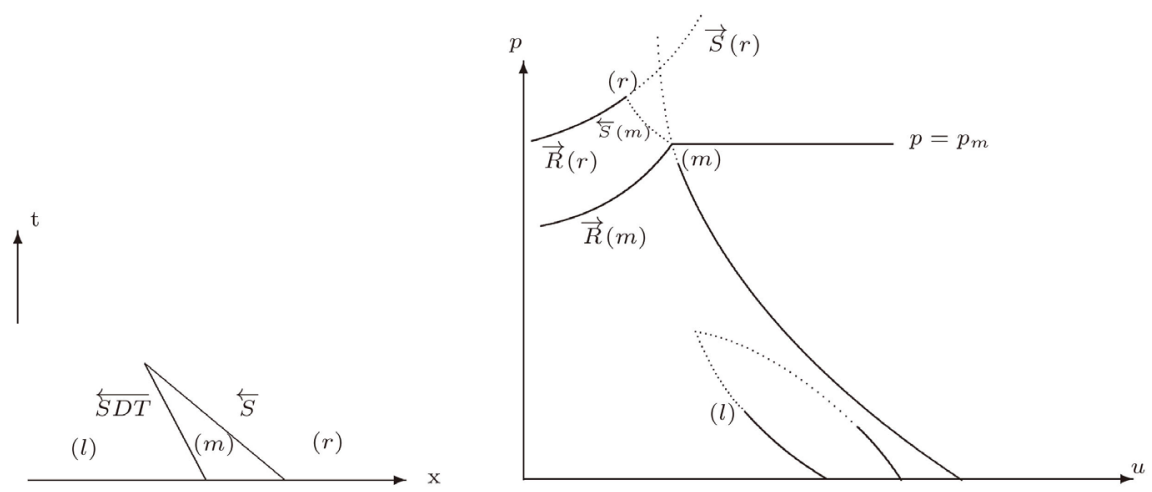

Figure 17. The interaction of $\overline{S D T}$ and $\bar{S}$.

straight line $p=p_{m}\left(u>u_{m}\right)$. Therefore, $\vec{W}_{S}(r)$ does not intersect with $\bar{W}_{S}(l) \cup \bar{W}_{D F}(l)$ and the result of the interaction is expressed by

$$
\overline{S D T} \bar{S} \rightarrow \begin{cases}\overline{S D T}+\vec{R} \text { or } \vec{S}, & p_{l} \tau_{l}^{\gamma}=p_{r} \tau_{r}^{\gamma}, \\ \overline{S D T}+J+\vec{R} \text { or } \vec{S}, & p_{l} \tau_{l}^{\gamma} \neq p_{r} \tau_{r}^{\gamma} .\end{cases}
$$

Theorem 3.6. For this case, when a shock wave overtakes a strong detonation combustion wave, it speeds up the strong detonation combustion wave and the contact discontinuity may appear or not.

\section{Case 6. $\overline{W D F} \bar{S}$}

In this case (Figure 18), similar with the discussions as the just above case, we know that $\bar{S}$ will overtake $\overline{S D T}$ at a finite time and a new Riemann problem is formed with $(I)$ and $(r)$ as its left-hand side state and right-hand side state, respectively. Notice that $(m) \in \bar{W}_{D F}(l),(r) \in \bar{S}(m)$ and $(r)$ is located above the curve $\bar{W}_{D F}(l)$, we know the temperature at this point is higher than the ignition temperature, therefore when $p_{l} \tau_{l}^{\gamma}=p_{r} \tau_{r}^{\gamma}$, the results is described by

$$
\overline{W D F} \bar{S} \rightarrow\left\{\begin{array}{l}
\overline{W D F}+\vec{R} \text { or } \vec{S}, \\
\vec{R} \text { or } \vec{S}, \\
\overline{D T}+\vec{R} \text { or } \vec{S},
\end{array}\right.
$$

when $p_{l} \tau_{l}^{\gamma} \neq p_{r} \tau_{r}^{\gamma}$, the result is the same expect that the contact discontinuity appears here.

Theorem 3.7. For this case, when a shock wave overtakes a weak deflagration combustion wave, the weak deflagration combustion wave may be extinguished or be transformed into a detonation wave. And the contact discontinuity may appear or not.

After the discussions of the elementary wave interactions, we summarize the results as follows.

By investigating the kinds of wave interactions of the elementary waves, we can capture some interesting combustion phenomena. For example, the combustion wave may be extinguished by the contact discontinuity or by the shock wave. Especially, a detonation wave may be transformed into a weak deflagration wave coalescing with pre-compression shock wave by the contact 


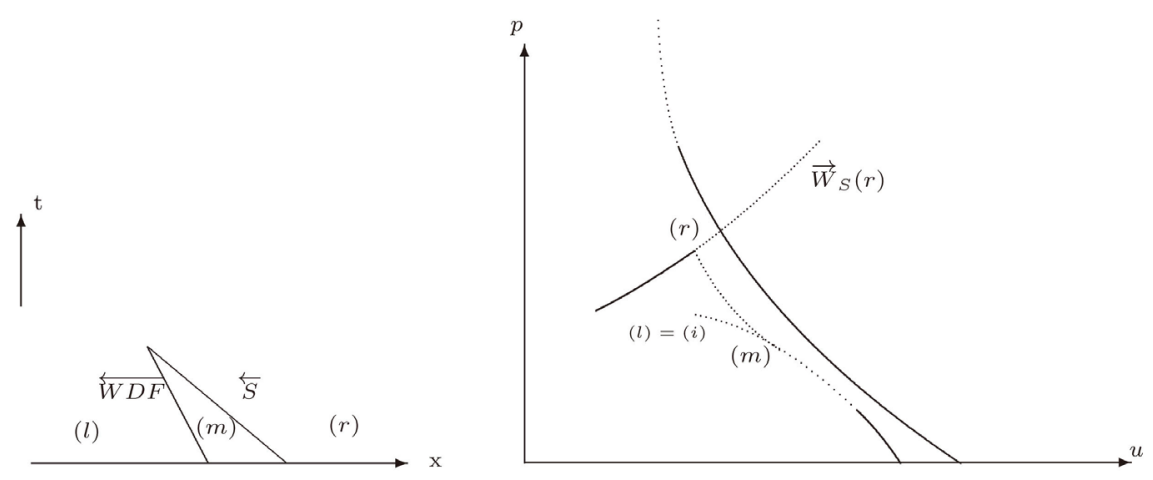

Figure 18. The interaction of $\overline{W D F}$ and $\bar{S}$.

discontinuity, and a deflagration wave may be transformed into a detonation wave by the contact discontinuity or by the shock wave, which describe the transition between the detonation wave and the deflagration wave. Notice specially that the contact discontinuity play a very important role in magnetogasdynamic combustion model (1) and (3), which is very different from the conventional gas dynamic combustion model (2) and (3).

Since the reaction rate in our model is infinite which is an idealized hypothesis, while our model is still very important in application, we will investigate the initial value problem for the self-similar Zeldovich-von Neumann-Döring (ZND) model in magnetogasdynamic combustion with finite reaction rate in our coming works.

\section{Supported}

Partially by the Foundation for Young Scholars of Shandong University of Technology (No. 115024).

\section{Conflicts of Interest}

The authors declare that there is no conflict of interest regarding the publication of this paper.

\section{References}

[1] Chen, G.Q. and Wang, D.H. (2002) Global Solutions of Nonlinear Magnetohydrodynamics with Large Initial Data. Journal of Differential Equations, 182, 344-376. https://doi.org/10.1006/jdeq.2001.4111

[2] Cowling, T.G. (1957) Magnetoydrodynamics. Interscience, New York.

[3] Helliwell, J.B. (1962) Gas-Ionizing Shock and Combustion Waves in Magnetogasdynamics. Journal of Fluid Mechanics, 14, 405-419. https://doi.org/10.1017/S0022112062001329

[4] Hu, Y.B. and Sheng, W.C. (2013) The Riemann Problem of Conservation Laws in Magnetogasdynamics. Communications on Pure and Applied Analysis, 12, 755-769. https://doi.org/10.3934/cpaa.2013.12.755

[5] Liu, Y.J. and Sun, W.H. (2019) The Riemann Problem for the Simplified Combustion Model in Magnetogasdynamics, Submitted. 
[6] Mareev, V.A. (1975) Self-Similar Magnetogasdynamic Flows Accompanied by detonation and Combustion Waves. Fluid Dynamics, 10, 86-93. https://doi.org/10.1007/BF01023785

[7] Pu, X.K. and Guo, B.L. (2013) Global Existence and Convergence Rates of Smooth Solutions for the Full Compressible MHD Equations. Zeitschrift für Angewandte Mathematik und Physik, 64, 519-538. https://doi.org/10.1007/s00033-012-0245-5

[8] Sekhar, T.R. and Sharma, V.D. (2010) Riemann Problem and Elementary Wave Interactions in Isentropic Magnetogasdynamics. Nonlinear Analysis: Real World Applications, 11, 619-636. https://doi.org/10.1016/j.nonrwa.2008.10.036

[9] Shen, C. (2011) The Limits of Riemann Solutions to the Isentropic Magnetogasdynamics. Applied Mathematics Letters, 24, 1124-1129.

https://doi.org/10.1016/j.aml.2011.01.038

[10] Courant, R. and Friedrichs, K.O. (1948) Supersonic Flow and Shock Waves. Interscience, New York.

[11] Liu, Y.J. and Sheng, W.C. (2011) The Generalized Riemann Problem for Gas Dynamic Combustion. Applied Mathematics and Mechanics (English Series), 32, 1079-1090. https://doi.org/10.1007/s10483-011-1482-6

[12] Sun, M.N. (2017) Singular Solutions to the Riemann Problem for a Macroscopic Production MODEL. Zeitschrift fur Angewandte Mathematik und Mechanik, 97, 916-931. https://doi.org/10.1002/zamm.201600171

[13] Zhang, T. and Zheng, Y.X. (1989) Riemann Problem for Gasdynamic Combustion. Journal of Differential Equations, 77, 203-230.

https://doi.org/10.1016/0022-0396(89)90142-3 